\title{
IgA1-secreting cell lines from patients with IgA nephropathy produce aberrantly glycosylated IgA1
}

\author{
Hitoshi Suzuki, ${ }^{1,2}$ Zina Moldoveanu, ${ }^{1}$ Stacy Hall, ${ }^{1}$ Rhubell Brown, ${ }^{1}$ Huong L. Vu, ${ }^{1}$ Lea Novak, ${ }^{1}$ \\ Bruce A. Julian, ${ }^{1}$ Milan Tomana, ${ }^{1}$ Robert J. Wyatt, ${ }^{3}$ Jeffrey C. Edberg, ${ }^{1}$ Graciela S. Alarcón, ${ }^{1}$ \\ Robert P. Kimberly, ${ }^{1}$ Yasuhiko Tomino, ${ }^{2}$ Jiri Mestecky, ${ }^{1,4}$ and Jan Novak ${ }^{1}$ \\ ${ }^{1}$ University of Alabama at Birmingham, Birmingham, Alabama, USA. 2Juntendo University School of Medicine, Tokyo, Japan. \\ ${ }^{3}$ University of Tennessee Health Science Center, Memphis, Tennessee, USA. ${ }^{4}$ Charles University, School of Medicine, Prague, Czech Republic.
}

\begin{abstract}
Aberrant glycosylation of IgA1 plays an essential role in the pathogenesis of IgA nephropathy. This abnormality is manifested by a deficiency of galactose in the hinge-region $O$-linked glycans of IgA1. Biosynthesis of these glycans occurs in a stepwise fashion beginning with the addition of $N$-acetylgalactosamine by the enzyme $\mathrm{N}$-acetylgalactosaminyltransferase 2 and continuing with the addition of either galactose by $\beta 1,3$-galactosyltransferase or a terminal sialic acid by a $N$-acetylgalactosamine-specific $\alpha 2,6$-sialyltransferase. To identify the molecular basis for the aberrant IgA glycosylation, we established EBV-immortalized IgA1-producing cells from peripheral blood cells of patients with IgA nephropathy. The secreted IgA1 was mostly polymeric and had galactose-deficient $O$-linked glycans, characterized by a terminal or sialylated $N$-acetylgalactosamine. As controls, we showed that EBV-immortalized cells from patients with lupus nephritis and healthy individuals did not produce IgA with the defective galactosylation pattern. Analysis of the biosynthetic pathways in cloned EBV-immortalized cells from patients with IgA nephropathy indicated a decrease in $\beta 1,3$-galactosyltransferase activity and an increase in $\mathrm{N}$-acetylgalactosamine-specific $\alpha 2,6$-sialyltransferase activity. Also, expression of $\beta 1,3$-galactosyltransferase was significantly lower, and that of $N$-acetylgalactosamine-specific $\alpha 2,6$-sialyltransferase was significantly higher than the expression of these genes in the control cells. Thus, our data suggest that premature sialylation likely contributes to the aberrant IgA1 glycosylation in IgA nephropathy and may represent a new therapeutic target.
\end{abstract}

\section{Introduction}

IgA nephropathy (IgAN) is the most common primary glomerulonephritis worldwide (1), with about $20 \%-40 \%$ of patients developing end-stage renal failure. IgAN is defined by the dominance or codominance of IgA in the mesangial immune deposits $(2,3)$. There is now considerable evidence that the mesangial immune deposits originate from circulating IgA1-containing immune complexes (4-10). The IgA1 in these complexes $(11,12)$ and in the glomerular deposits of IgAN patients $(5,6)$ is aberrantly glycosylated, i.e., the hinge-region $O$-linked glycans are galactose-deficient (Gal-deficient) $(7,8,10)$. The absence of Gal apparently leads to the exposure of epitopes that are normally sequestered, including terminal and sialylated $N$-acetylgalactosamine (GalNAc) residues (9). These epitopes are recognized by naturally occurring IgG or IgA1 antibodies with anti-glycan specificities and, consequently, circulating immune complexes are formed (12). The clearance of the Gal-deficient IgA1 in these immune complexes is delayed, promoting its deposition in the glomeruli $(9,13)$. Experiments in vitro have established that binding of the immune complexes con-

Nonstandard abbreviations used: BADG, benzyl 2-acetamido-2-deoxy- $\alpha$-D-galactopyranoside; C1GalT1, core $1 \beta 1,3$ galactosyltransferase; Cosmc, C1GalT1-specific molecular chaperone; Gal, galactose; GalNAc, $N$-acetylgalactosamine; GalNAcT2, $\mathrm{N}$-acetylgalactosaminyltransferase 2; HAA, Helix aspersa lectin; HC, healthy control; IgAN, IgA nephropathy; MES, 2-[N-Morpholino]ethanesulfonic acid; NeuAc, $N$-acetylneuraminic acid; SNA, Sambucus nigra lectin; ST6GalNAc, $\alpha 2,6$-GalNAcsialyltransferase; UDP, uridine-5'-disphospho.

Conflict of interest: The authors have declared that no conflict of interest exists. Citation for this article: J. Clin. Invest. 118:629-639 (2008). doi:10.1172/JCI33189. taining aberrantly glycosylated IgA1 to the mesangial cells induces pathologic changes that are characteristic of IgAN (13-22). Taken together, these findings indicate that aberrantly glycosylated IgA1 plays a pivotal role in the pathogenesis of IgAN.

In humans, each IgA1 heavy chain hinge region has 3 to 5 $O$-linked glycans that are built by stepwise addition of monosaccharides: GalNAc is linked to the serine and threonine in the IgA1 hinge region by the enzyme $N$-acetylgalactosaminyltransferase 2 (GalNAcT2) (23), Gal is attached to GalNAc by core $1 \beta 1,3$-galactosyltransferase (C1GalT1) (24) in association with its chaperone, Cosmc $(25,26)$, and sialic acid $N$-acetylneuraminic acid (NeuAc) may be linked to GalNAc by $\alpha 2,6$-GalNAc-sialyltransferase I or II (ST6GalNAcI or ST6GalNAcII) (27-29). NeuAc can be attached also to Gal by an $\alpha 2,3-$ Gal-sialyltransferase $(30,31)$. Theoretically, the Gal deficiency of IgA1 O-linked glycans in IgAN could occur as a result of elevated activity of ST6GalNAc, as sialylation of GalNAc blocks its galactosylation (32), or reduced activity of C1GalT1, or it could result from a combination of both of these defects (10).

An understanding of the molecular basis for the variations in the carbohydrate content of IgA1 in IgAN and the identification of the roles of individual glycosyltransferases in these events is essential for the definition of the fundamental defects that result in the synthesis of the aberrant glycans in IgAN. Such studies have been stymied, however, by the lack of experimental models. IgA1 is present exclusively in humans and hominoid primates $(33,34)$, which suggests that the use of small-animal models may generate results irrelevant to IgAN in humans; furthermore, it is difficult to obtain sufficient cells of interest from human mucosal tissues, lymph nodes, or bone 


\section{Table 1}

Clinical characteristics of the study population

\begin{tabular}{|c|c|c|c|c|c|}
\hline & $\begin{array}{c}\text { Age } \\
\text { (years) }\end{array}$ & $\begin{array}{l}\text { Serum creatinine } \\
(\mathrm{mg} / \mathrm{dl})\end{array}$ & $\begin{array}{l}\mathrm{UP} / \mathrm{Cr} \\
\text { ratio }^{A}\end{array}$ & $\mathrm{U} \lg \mathrm{A} / \mathrm{Cr}^{\mathrm{B}}$ & U HAA-IgA1/CrC \\
\hline IgAN patients $(n=11)$ & $41.4 \pm 19.0$ & $1.7 \pm 1.2$ & $1.3 \pm 1.1$ & $155.0 \pm 187.7$ & $17.1 \pm 8.8$ \\
\hline Healthy controls $(n=11)$ & $44.9 \pm 17.6$ & $0.9 \pm 0.2$ & $0.1 \pm 0.1$ & $3.3 \pm 1.9$ & $6.2 \pm 2.3$ \\
\hline Disease controls (lupus nephritis) $(n=10)$ & $38.1 \pm 12.1$ & $1.1 \pm 0.3$ & $4.1 \pm 5.3$ & ND & ND \\
\hline
\end{tabular}

Data expressed as mean \pm SD. AUrinary protein/creatinine ratio. ${ }^{B}$ Levels of urinary lgA relative to urinary creatinine. ${ }^{C}$ Levels of urinary Gal-deficient IgA1 (HAA-IgA1) relative to urinary creatinine. ND, not done.

marrow. We therefore explored the possibility that EBV-immortalized cell lines established from circulating IgA1-producing B cells would prove to be a suitable model for such analyses. Analysis of EBV-immortalized B cells from patients with IgAN, patients with biopsy-proven lupus nephritis, and healthy control individuals indicated that these IgA1-secreting cell lines retained the phenotype of the circulating IgA1-secreting cells. Thus, the EBV-immortalized IgA1-secreting cells from patients with IgAN secreted IgA1 with Galdeficient $O$-linked glycans, and the IgA1 was predominantly in the polymeric form, whereas the IgA1-secreting EBV-transformed B cells from patients with biopsy-proven lupus nephritis or from healthy individuals secreted IgA1 that was normally glycosylated. In all cases, the degree of Gal deficiency in O-glycans on IgA1 in the cell supernatants was equivalent to that on IgA1 in the serum. Cloned EBVimmortalized IgA1-secreting cell lines from patients with IgAN, here referred to as IgAN-IgA1S cell lines, and healthy control individuals, here referred to as HC-IgA1S cell lines, were established. All of the IgAN-IgA1S cell lines were found to exhibit significant differences in the expression of C1GalT1 and ST6GalNACII, and the activities of the corresponding enzymes, as compared with the HC-IgA1S cell lines or the EBV-immortalized IgA1-secreting cells from the patients with lupus nephritis. Collectively, our findings suggest that "premature" sialylation contributes to the aberrant IgA1 glycosylation in IgAN.

\section{Results}

Generation of immunoglobulin-secreting EBV-immortalized cells from PBMCs of patients with IgAN or lupus nephritis and healthy controls. PBMCs obtained from 57 patients with IgAN, 10 patients with lupus nephritis, and 63 healthy controls were immortalized with EBV. The immunoglobulin levels secreted by the EBV-immortalized cells from patients with IgAN or lupus nephritis and healthy controls were similar $(4.30 \pm 5.22,3.87 \pm 1.05$, and $4.55 \pm 5.86 \mu \mathrm{g} / \mathrm{ml}$ $\mathrm{IgA} ; 12.47 \pm 13.66,13.14 \pm 0.84$, and $14.50 \pm 10.94 \mu \mathrm{g} / \mathrm{ml} \mathrm{IgG}$; and $20.41 \pm 27.02,15.78 \pm 14.02$, and $19.74 \pm 16.67 \mu \mathrm{g} / \mathrm{ml} \mathrm{IgM}$,

\section{Figure 1}

IgA1 secreted by IgA1-producing cell lines from patients with IgAN is Gal deficient. (A) Degree of Gal deficiency of $O$-glycans on IgA1 secreted by IgAN-IgA1S cell lines ( $n=11$; filled circles) and HC-IgA1S cell lines ( $n=11$; open circles) was measured by lectin ELISA. The results were calculated relative to HAA reactivity of the standard naturally Gal-deficient IgA1 (Mce) myeloma protein (its relative HAA reactivity is set to $100 \%)$. IgA1 secreted by the $\lg A N-\lg A 1 S$ cell lines showed higher reactivity with HAA than that secreted by the HC-IgA1S cell lines $(P=0.0001)$. (B) Serum levels of Gal-deficient IgA1 correlate with $\mathrm{Gal}$ deficiency of $\lg \mathrm{A} 1$ secreted by the corresponding cell lines $\left(P<0.001 ; \mathrm{R}^{2}=0.883\right)$. The mean values were expressed relative to HAA reactivity of the standard Gal-deficient IgA1 (Mce) myeloma protein, as in $\mathbf{A}$. respectively). The IgA1 secreted by the cells established from IgAN patients was Gal-deficient in the $O$-linked glycans as estimated by reactivity with the GalNAc-specific lectin from Helix aspersa (HAA) (35). The normalized HAA reactivity (values normalized to total IgA and expressed as percentage of the standard Gal-deficient IgA1 [Mce] myeloma protein) was $27.48 \% \pm 10.67 \%$ for IgA1 secreted by cells established from IgAN patients, $13.05 \% \pm 3.43 \%$ for IgA1 secreted by cells established from patients with lupus nephritis $(P=0.0007)$, and $12.63 \% \pm 8.13 \%$ for IgA1 secreted by cells established from healthy controls $(P<0.0001)$. There was no difference for the values between healthy controls (serum creatinine ranging from 0.7 to $1.1 \mathrm{mg} / \mathrm{dl}$ ) and patients with lupus nephritis (serum creatinine ranging from 0.8 to $1.7 \mathrm{mg} / \mathrm{dl}$ ). Thus, only the immortalized cells from IgAN patients produced Gal-deficient IgA1.

We then subcloned the EBV-immortalized IgA-secreting cell lines from 11 randomly selected patients with IgAN and 11 randomly selected healthy controls by limiting dilution (Table 1 ). The resultant cell lines secreted $6.6 \pm 3.1$ and $6.7 \pm 2.7 \mu \mathrm{g} / \mathrm{ml}$ IgA, respectively, but no IgG or IgM. Based on immunofluorescence analysis using IgA1and IgA2-specific monoclonal antibodies, all of the cell lines secreted exclusively IgA1. The IgA1 secreted by the IgAN-IgA1S cell lines but

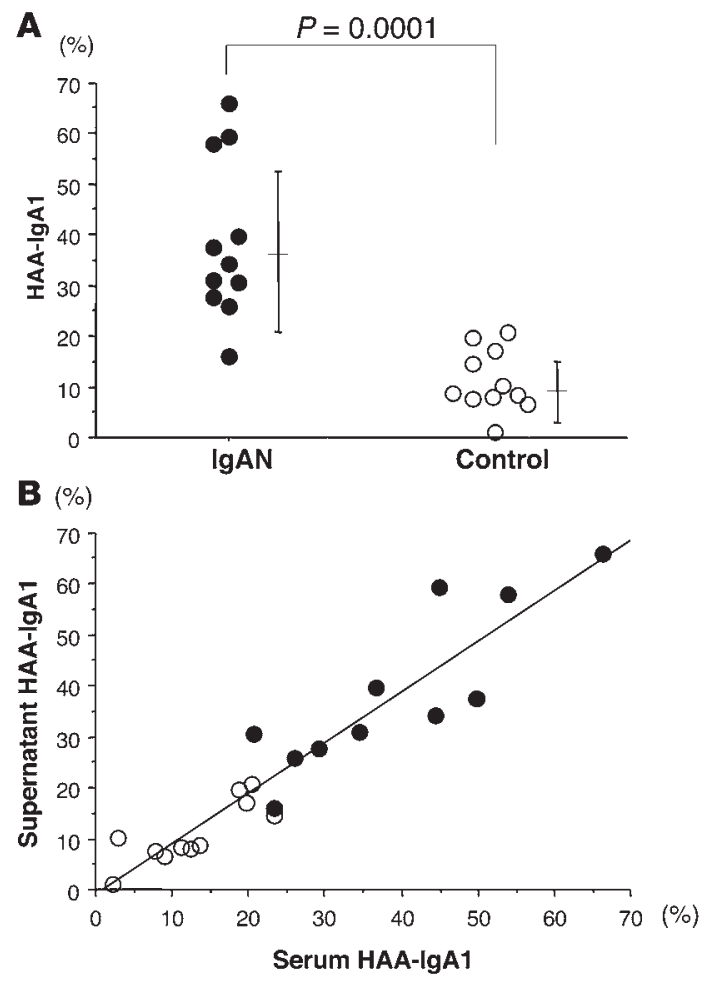


A

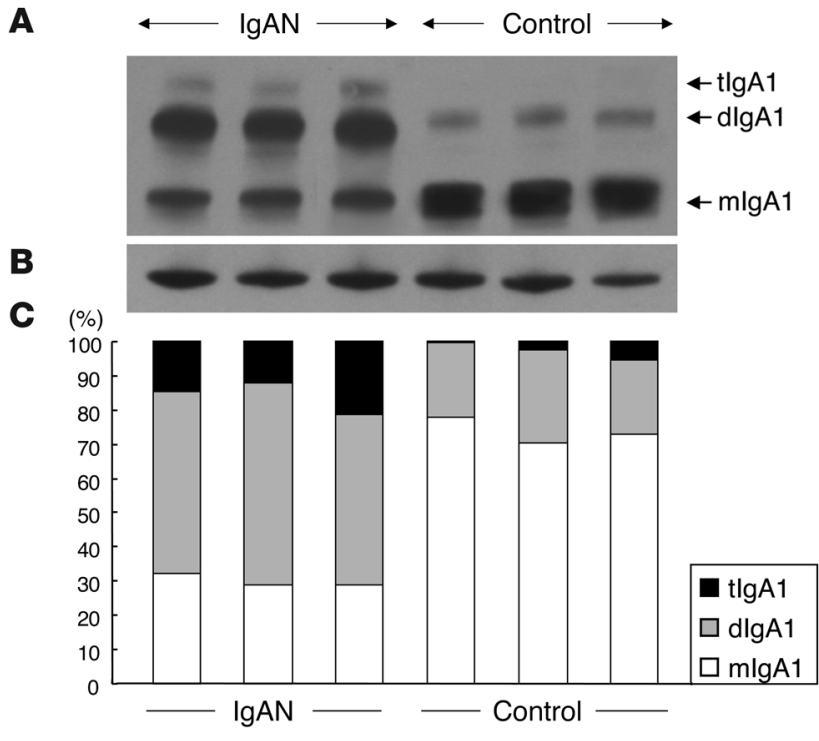

not the HC-IgA1S cell lines was Gal-deficient $(P=0.0001)$ (Figure 1A). Furthermore, the degree of Gal deficiency of the IgA1 in the culture supernatants was similar to that of the circulatory IgA1 in the serum samples from the donors $\left(\mathrm{R}^{2}=0.883 ; P<0.001\right)$ (Figure 1B).

Characterization of the molecular forms and glycosylation of IgA1 secreted by the cell lines established from PBMCs from IgAN patients and healthy controls. Three IgAN-IgA1S cell lines and 3 HC-IgA1S cell lines, all of which were established using PBMCs from different donors, were randomly selected for further analysis. The secreted IgA1 was first analyzed by Western blotting after SDS-PAGE separation under nonreducing conditions (Figure 2A). Densitometric analysis of these blots, normalized to the total amount of IgA1 heavy chain, revealed that all of the tested IgAN-IgA1S cell lines secreted predominantly dimeric and trimeric IgA1 (Figure $2 \mathrm{~B}$ ), whereas all of the tested HC-IgA1S cell lines secreted mostly monomeric IgA1 (Figure 2C). Size-exclusion chromatography of the cell-culture supernatants from 3 IgAN-IgA1S and 2 HC-IgA1S cell lines confirmed these findings (Figure 3A). Furthermore, a lectin ELISA showed that the dimeric and trimeric IgA1 secreted by the IgANIgA1S cell lines were Gal-deficient (Figure 3B).

To determine whether the Gal-deficient $O$-linked glycans contained mostly terminal or sialylated GalNAc, we used a lectin ELISA (35) in which HAA specifically recognizes GalNAc in the untreated or neuraminidase-treated IgA1. The HAA reactivity of the IgA1 secreted by each of the IgAN-IgA1S cell lines was enhanced significantly by treatment with neuraminidase $(P<0.01)$ (Fig-

\section{Figure 3}

Analysis of $\lg A 1$ fractionated by size-exclusion chromatography. Culture supernatants from the 3 randomly selected IgAN-IgA1S cell lines (filled circles) and $2 \mathrm{HC}-\lg A 1 \mathrm{~S}$ cell lines (open circles) were separated by size-exclusion chromatography. Concentration of IgA1 in the fractions was determined by ELISA (A). Western blotting analysis after SDS-PAGE separation under nonreducing conditions confirmed distinct molecular forms of $\lg A$ in various fractions (inset in $\mathbf{A}$ ). (B) Gal deficiency of IgA1 was determined by HAA-ELISA. Trimeric and dimeric IgA1 secreted by IgAN-IgA1S cell lines had high reactivity with HAA, while monomeric IgA1 did not react. Furthermore, IgA1 secreted by $\mathrm{HC}$-lgA1S cell lines was mostly monomeric and did not react with $\mathrm{HAA}$. Data are expressed as mean \pm SD.

\section{Figure 2}

Analysis of molecular form of IgA1 secreted by IgA1-producing cell lines. Supernatants from randomly selected cell lines from 3 IgAN patients and 3 healthy controls were separated by SDS-PAGE under nonreducing $(\mathbf{A})$ and reducing $(\mathbf{B})$ conditions. The loaded samples were normalized to total IgA content (10 ng/well). (C) Densities of the bands in $\mathbf{A}$ were quantitated by densitometry. Cell lines from IgAN patients secreted predominantly polymeric IgA1 (about $70 \%$ of total $\operatorname{lgA} 1$ was dimeric [d] and trimeric [t]) in contrast to only $20 \%-30 \%$ as polymeric forms for the IgA1 secreted by the cell lines from healthy controls. mlgA1, monomeric $\lg \mathrm{A} 1$.

ure 4A). Western blot analysis with HAA (Figure 4B) or Sambucus nigra lectin (SNA) (detects $\alpha 2,6$-bound NeuAc) (Figure 4C) confirmed excess sialylation of GalNAc on the Gal-deficient $O$-linked glycans of IgA1 from the IgAN-IgA1S cell lines.

Gal-deficient IgA1 is localized in the Golgi apparatus of cell lines producing the aberrant IgA1. The IgA1 in the cytoplasm of IgAN-IgA1S cell lines, but not HC-IgA1S cell lines, reacted with HAA after treatment of the cells with neuraminidase, indicating a Gal deficiency (Figure 5, A and B). Using confocal microscopy, we confirmed that the Gal-deficient IgA1 was localized in the Golgi apparatus (Figure 6A). The intensity of HAA staining of IgA1 in the Golgi apparatus was enhanced by neuraminidase treatment (Figure 6, A and B). Thus, the aberrantly glycosylated $O$-linked glycans of IgA1 were present throughout the Golgi system as sialylated GalNAc glycans.

Gene expression of specific glycosyltransferases in the IgA1-producing cells of patients with IgAN or lupus nephritis and healthy controls. Using realtime RT-PCR with SYBR Green I detection and specific primers for GalNAcT2, C1GalT1, Cosmc, ST6GalNAcI, -II, -IV, and -VI, IgA a chain, and $J$ chain (Table 2), we determined the gene expression in the IgANIgA1S and HC-IgA1S cell lines. The mean levels of transcripts for

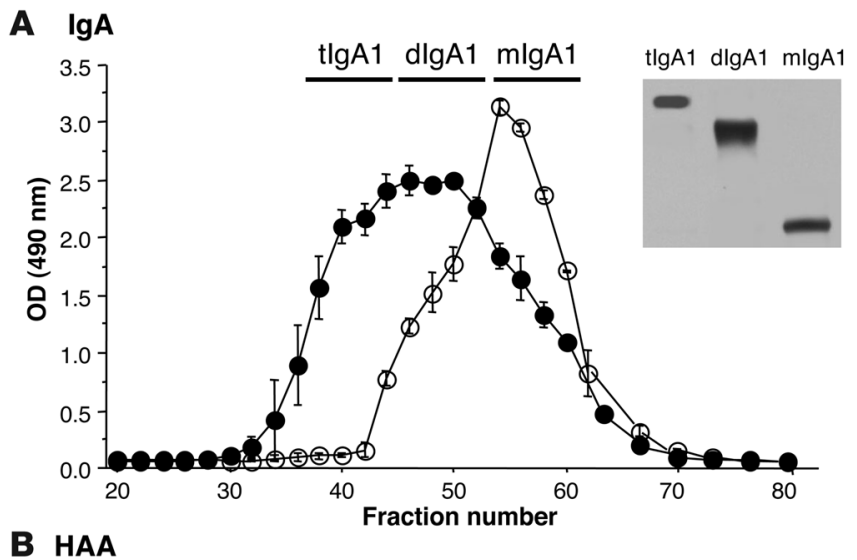

B HAA

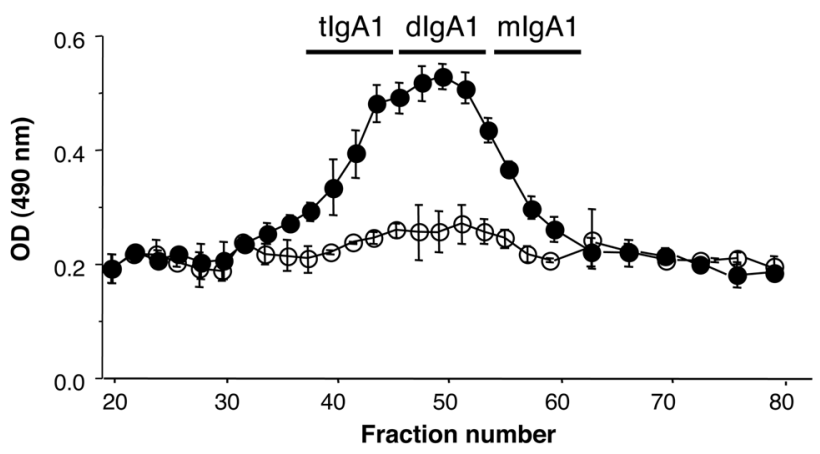



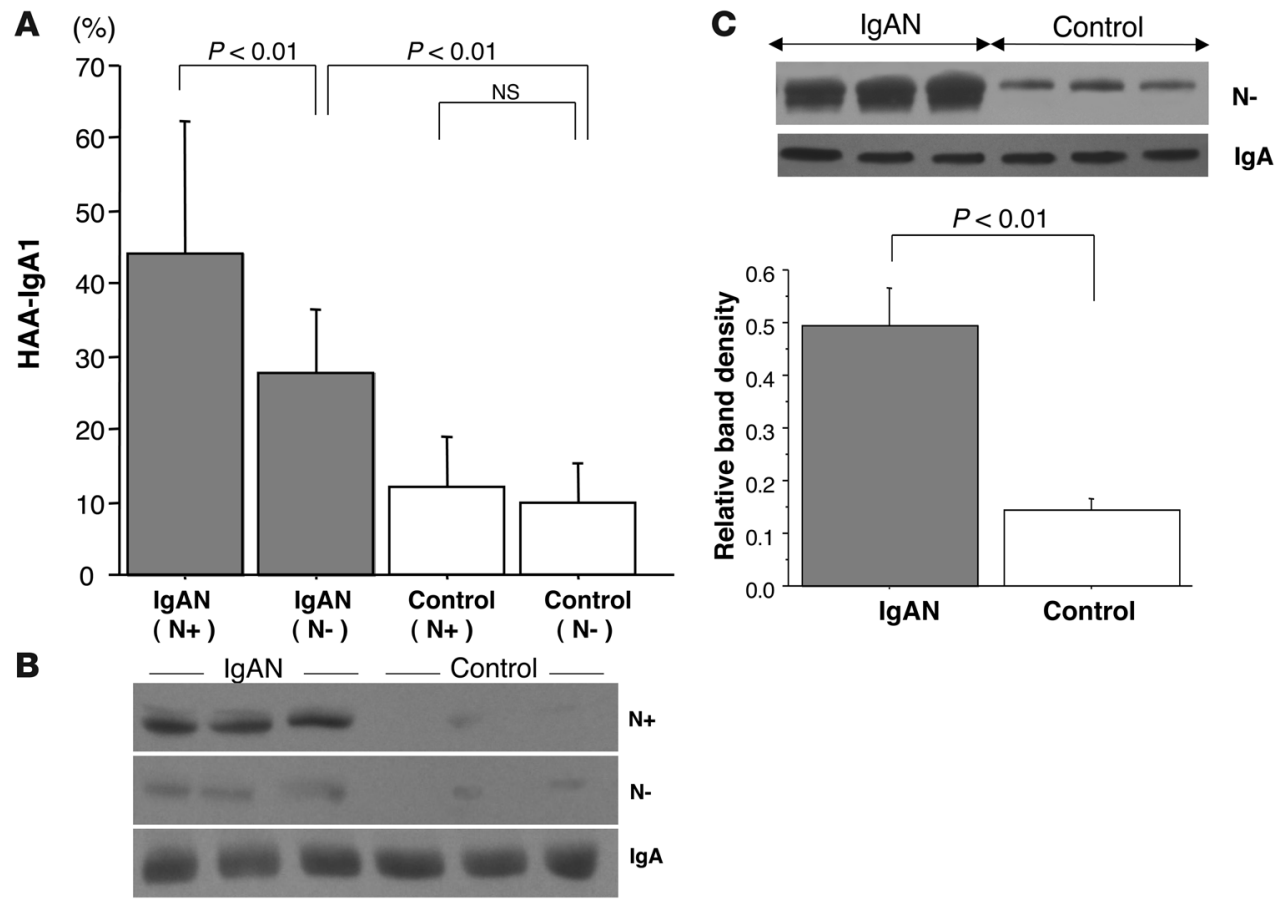

\section{Figure 4}

IgA1 secreted by cell lines from IgAN patients has Gal-deficient $O$-linked glycans with terminal or sialylated GalNAc. (A) Gal-deficient IgA1 was measured by HAA-ELISA as the ratio of HAA binding $\lg A 1$ to total $\lg A 1$ with $(\mathrm{N}+)$ or without $(\mathrm{N}-)$ neuraminidase treatment. The values were expressed relative to HAA reactivity of the standard Gal-deficient IgA1 (Mce) myeloma protein, as described in Figure 1. Reactivity of IgA1 with HAA increased after neuraminidase treatment of IgA1 from cell lines from 5 IgAN patients but not from 5 healthy controls. Western blots obtained after SDS-PAGE under reducing conditions were developed with HAA before $(\mathrm{N}-)$ or after $(\mathrm{N}+)$ neuraminidase treatment $(B)$ or with SNA $(\alpha 2,6-N e u A c-s p e c i f i c ~ l e c t i n)(C)$. The loaded samples were normalized to total lgA (10 ng/well) (load control; lower panels developed with lgA-specific antibody). IgA1 secreted by IgAN-lgA1S cell lines reacted with HAA, and this reactivity was enhanced by neuraminidase treatment; in contrast, the IgA1 secreted by HC-IgA1S cell lines only marginally reacted with HAA (B). (C) SNA Western blotting of IgA1 not treated with neuraminidase. The results confirmed that the IgA1 secreted by IgAN-IgA1S cell lines was highly sialylated, whereas the IgA1 secreted by HC-IgA1S cell lines was less sialylated. Bands were densitometrically quantified and expressed as ratio of SNA-binding IgA to total IgA (bar graph shows mean \pm SD).

both C1GalT1 and its molecular chaperone Cosmc were significantly lower in the IgAN-IgA1S cell lines than in the HC-IgA1S cell lines $(P=0.0004$ and $P=0.0001$, respectively) (Figure 7A). ST6GalNAcI was not detected in either the IgAN-IgA1S or HC-IgA1S cell lines. Notably, the mean expression of ST6GalNAcII was significantly higher in the IgAN-IgA1S cell lines than in the HC-IgA1S cell lines $(P=0.0013)$ (Figure 7A). In contrast, the levels of GalNAcT2 (Figure 7A) and ST6GalNAcIV and ST6GalNAcVI (data not shown) were detected at equivalent levels in the IgAN-IgA1S and HC-IgA1S cell lines, as were IgA $\alpha$ chain and $J$ chain (Figure 7B). Analysis of the expression of C1GalT1, Cosmc, and ST6GalNAcII genes in EBV-immortalized IgAproducing cells from patients with lupus nephritis indicated that these were equivalent to those in similarly prepared cells from healthy controls (data not shown). Thus, only the cells from IgAN patients exhibited aberrant expression of C1GalT1, Cosmc, and ST6GalNAcII.

Enzymatic activities of $\beta 1,3$-galactosyltransferase and ST6GalNAc in IgA1-producing cell lines from IgAN patients and healthy controls. Using the Golgi-enriched fraction of the IgA1-producing cell lines as the enzyme source, desialylated IgA1 (Mce) myeloma protein as the acceptor, and an appropriate sugar donor (either uridine5 '-diphospho-Gal [UDP-Gal] for $\beta 1,3$-galactosyltransferase or CMP-NeuAc for ST6GalNAc), we measured the activities of $\beta 1,3$ galactosyltransferase and ST6GalNAc. The IgAN-IgA1S cell lines exhibited significantly lower $\beta 1,3$-galactosyltransferase activity
$(28.15 \pm 14.40 \mathrm{U} / \mathrm{mg}$ protein) than did the HC-IgA1S cell lines $(107.31 \pm 17.74 \mathrm{U} / \mathrm{mg}$ protein $)(P=0.006)$ (Figure $8 \mathrm{~A})$. The identity of the reaction product, and thus the specificity of the reaction, was confirmed by Western blot developed with HAA (Figure 8B). In contrast, the ST6GalNAc activity was several-fold higher in the IgAN-IgA1S cell lines $(97.84 \pm 32.37 \mathrm{U} / \mathrm{mg}$ protein $)$ than in the HC-IgA1S cell lines $(34.79 \pm 13.68 \mathrm{U} / \mathrm{mg}$ protein $)(P=0.011)$ (Figure $8 \mathrm{C}$ ). The identity of the reaction product, sialylated GalNAc, and thus the specificity of the reaction, was confirmed by HAA Western blotting of the untreated and neuraminidase-treated reaction product with HAA (Figure 8D). The increase in HAA binding of the IgA $1 \alpha$ chain after neuraminidase treatment was consistent with the neuraminidase-sensitive covering of GalNAc by NeuAc.

Inhibition of $\beta 1,3$-galactosyltransferase enhances the Gal deficiency of IgA1 secreted by cell lines established from PBMCs from IgAN patients and healthy controls. To confirm that the $\beta 1,3$-galactosyltransferase is functional in the IgA1-producing cell lines, we treated the cells with $5 \mathrm{mM}$ benzyl 2-acetamido-2-deoxy- $\alpha$-D-galactopyranoside (BADG) (36), a specific inhibitor of the enzyme. As measured by lectin ELISA, the proportion of Gal-deficient IgA1 secreted by IgAN-IgA1S as well as HC-IgA1S cell lines was greater in the treated cells than in the untreated cells $(P<0.01)$ (Table 3$)$. The IgA1 secreted by the BADGtreated HC-IgA1S cell lines bound HAA to the same degree, irrespective of neuraminidase treatment, a finding that is consistent with 
A
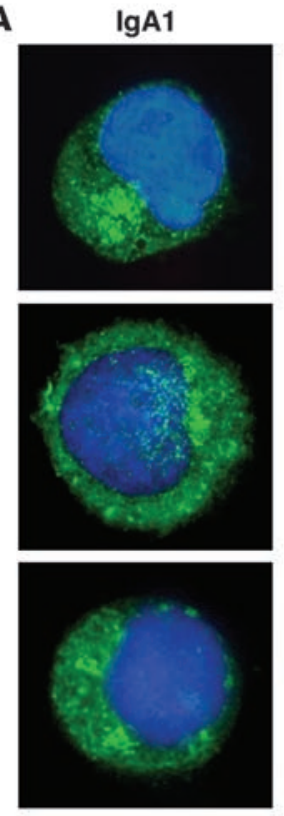

HAA, $\mathrm{N+}$
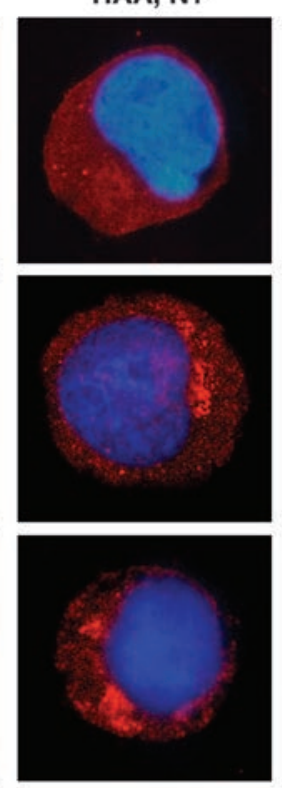
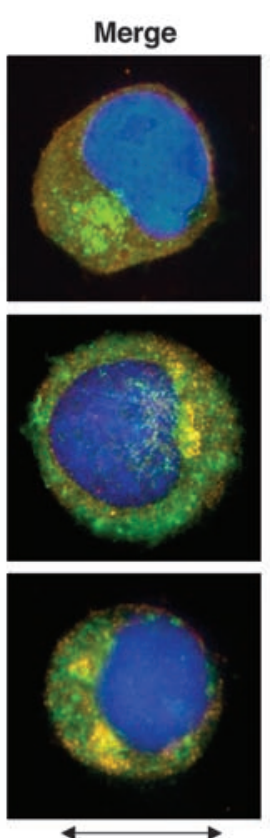

B
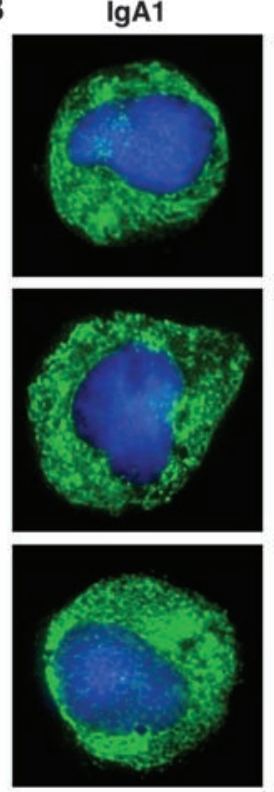

HAA, N+
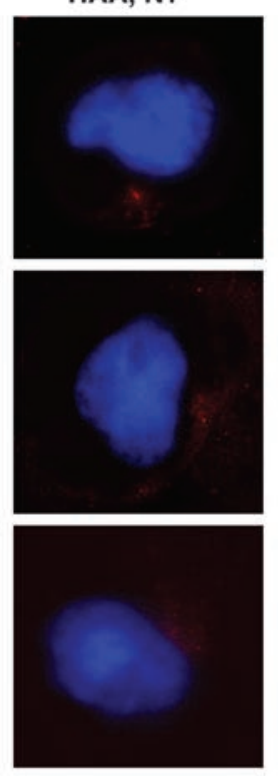
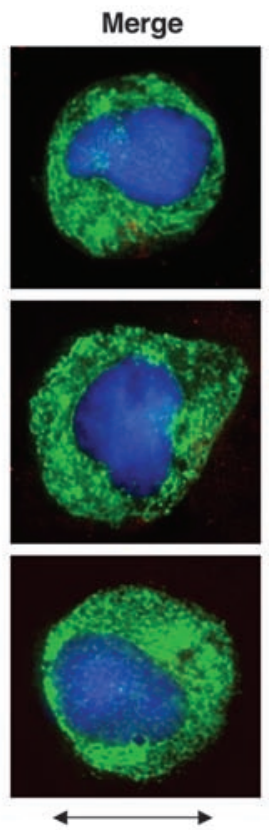

Figure 5

Immunofluorescence analysis of IgA1-producing cell lines. (A) HAA lectin bound to neuraminidase-treated IgA1 in the cytoplasm of cells secreting Gal-deficient IgA1 (IgAN-IgA1S cell lines from 3 IgAN patients). (B) IgA1 in HC-lgA1S cell lines from 3 healthy controls did not react with HAA, even after treatment with neuraminidase to remove terminal sialic acid residues. Staining for nuclei is shown in blue; for IgA, in green; and for HAA, in red. $\mathrm{N}_{+}$, neuraminidase treated. Scale bars: $10 \mu \mathrm{m}$.

the lack of NeuAc bound to GalNAc. In contrast, HAA binding to IgA1 secreted by IgAN-IgA1S cell lines was enhanced significantly by neuraminidase treatment, suggesting that many GalNAc residues (>40\%) were covered with NeuAc. These results confirmed the excess sialylation of GalNAc in the IgA1 secreted by IgAN-IgA1S cell lines and indicated that this occurred regardless of the inhibition of $\beta 1,3$-galactosyltransferase. Compared with the HC-IgA1S cell lines, the IgAN-IgA1S cell lines had less functional $\beta 1,3$-galactosyltransferase activity together with elevated sialyltransferase activity, leading to synthesis of Gal-deficient IgA1 with sialylated GalNAc.

\section{Discussion}

The lack of a full understanding of the etiopathogenesis of IgAN has hindered the development of a disease-specific therapy. There is a consensus opinion that aberrant glycosylation of IgA1 (Gal deficiency in the hinge-region $O$-linked glycans) is involved directly in the pathogenesis of IgAN $(7-9,37)$. The mechanisms leading to this aberrant glycosylation have not been studied extensively, however, due to the fact that expression of IgA1 is restricted to humans and hominoid primates. The lack of suitable animal models has necessitated new approaches for the development of a novel model that can be used to elucidate the molecular mechanisms that contribute to the development of this disease. In this study, we explored the possibility that EBV-immortalized cells from patients with IgAN could be used as such a model. We subcloned IgA1-secreting cell lines established from EBV-immortalized PBMCs of patients with IgAN and from healthy controls and characterized the glycosylation and molecular forms of the secreted immunoglobulin.

The EBV immortalization of the IgA1-producing cells did not affect the degree of Gal deficiency on $O$-glycans of IgA1 produced by the IgA1-secreting cells from patients with IgAN in terms of comparison with the IgA1 in the serum of these patients. Moreover, the IgA1 secreted by EBV-immortalized cells from both healthy and disease controls exhibited a normal Gal content in the $O$-glycans. The aberrantly glycosylated IgA1 secreted by the IgANIgA1S cell lines contained hinge-region glycans with sialylated or terminal GalNAc. This IgA1 was mostly polymeric, compared with the predominantly monomeric IgA1 secreted by the HC-IgA1S cell lines. In previous studies, the proportions of polymeric and monomeric IgA produced by lymphoblastoid IgA-secreting cell lines were found to vary, whereas the intracellular IgA was mostly monomeric (38). This result led the authors to speculate that the ability of the cells to produce polymers was dependent on the cellular content of $J$ chain and the stage of cellular maturation and differentiation. In our studies, we did not observe a difference in the mean expression levels of J chain between the IgAN-IgA1S and HC-IgA1S cell lines. This finding would favor the concept that the discrepancies in the production of the molecular forms may be related to the differentiation of the cell lines and/or distinctive genetic properties rather than the expression of the J chain. The IgA1 secreted by the IgAN-IgA1S cell lines reacted strongly with HAA. This binding was significantly enhanced by treatment with neuraminidase, suggesting that a substantial fraction of the Galdeficient glycans contained sialylated GalNAc. In the HC-IgA1S cell lines, the Gal content of the $O$-linked glycans of the hinge region approximated the full complement. Using SNA, a lectin specific for $\alpha 2,6-\mathrm{NeuAc}$, we confirmed an increased content of NeuAc in the hinge region of the IgA1 from the IgAN-IgA1S cell lines. The degree of Gal deficiency of the IgA1 secreted by the cell lines was equivalent to the Gal deficiency exhibited by the IgA1 isolated from the serum of the corresponding patient. Furthermore, a similar Gal deficiency and sialylation of GalNAc had been 
A $\lg A 1$
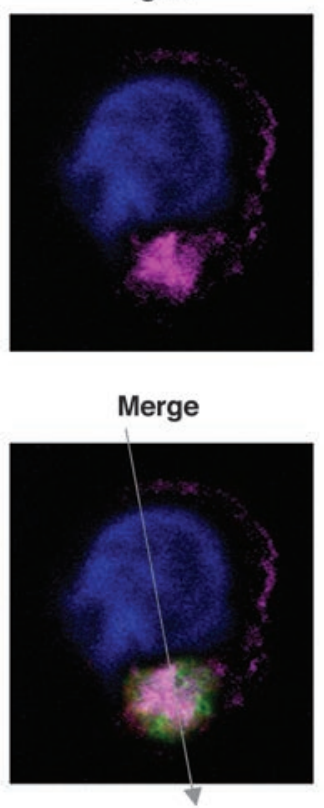

B
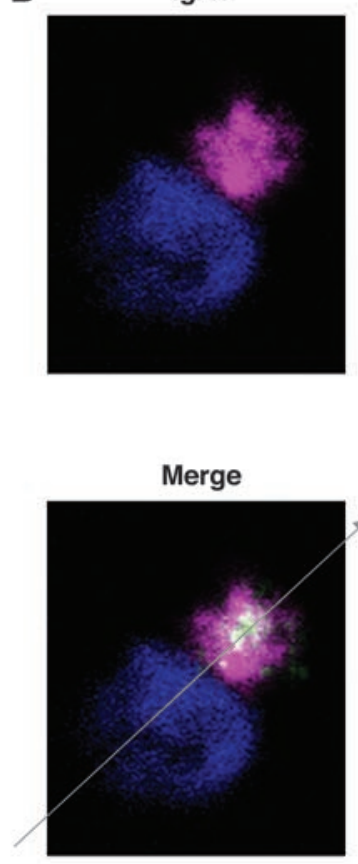

HAA, N+
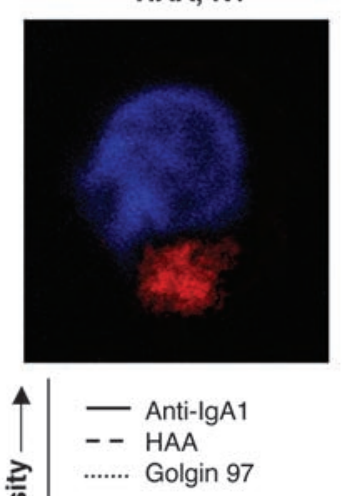
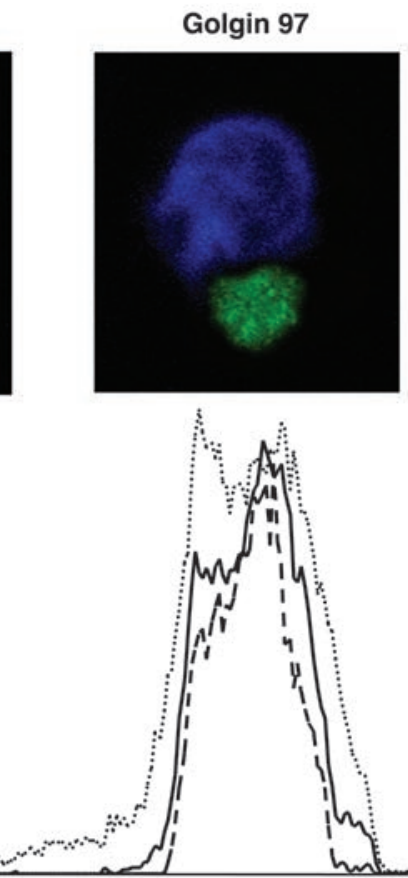

Analyzed line

HAA, N-
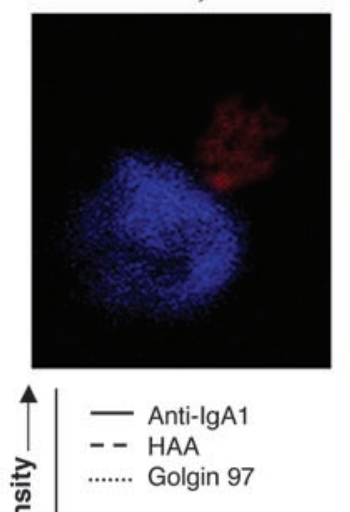

Golgin 97
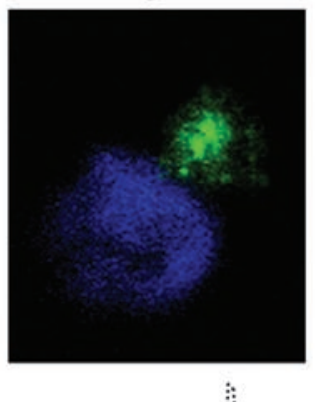

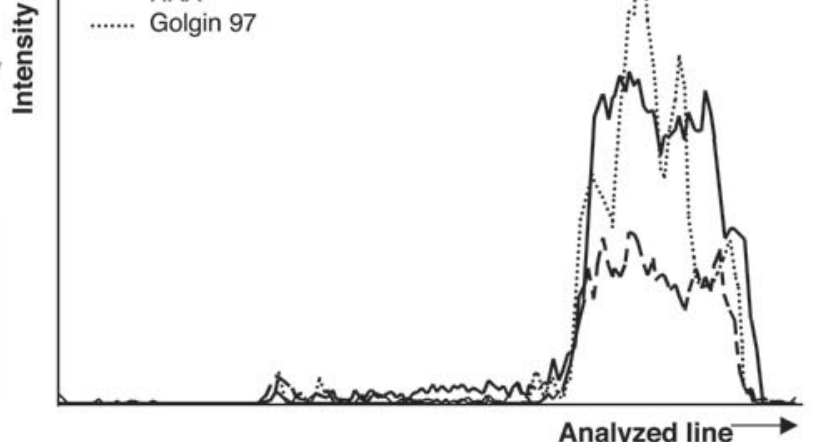

Analyzed line

\section{Figure 6}

HAA bound to Gal-deficient IgA1 in the Golgi apparatus. (A) Representative example of immunostaining with antihuman IgA antibody (violet), HAA lectin (after neuraminidase treatment) (red), and Golgi marker (Golgin 97) antibody (green) in cell from IgAN-IgA1S cell lines. IgA and HAA were colocalized in the Golgi apparatus. Fluorescence intensity profile from the confocal microscope is shown in the right-hand bottom panel (left bottom panel: line with arrow marks the cross section). (B) Same staining, but without neuraminidase treatment. Nuclear staining is shown in blue. $\mathrm{N}+$, neuraminidase treated; $\mathrm{N}-$, not treated with neuraminidase. reported for $O$-glycans of serum IgA1 from $\operatorname{IgAN}$ patients $(11,12$, 39-41). Thus, the cell lines established using PBMCs from IgAN patients produce IgA1 with all of the characteristics of the aberrant IgA1 typical of IgAN, including the quantitative rather than absolute character of differences in the Gal deficiency.

The apparent excess sialylation of the Gal-deficient $O$-linked glycans on IgA1 from IgAN patients implies potential pathogenic roles for several specific glycosyltransferases (10). It has been suggested that B cells in the circulation of IgAN patients have lower expression and/or show less activity of $\beta 1,3$-galactosyltransferase than such cells in healthy controls $(42,43)$. The stability and activity of this enzyme depends on a chaperone, Cosmc (25). Transcription of Cosmc has also been found to be reduced in IgAN patients (43). Mutations of this protein result in the production of Galdeficient $O$-linked glycans in Tn syndrome, a rare disease formerly called permanent mixed-field polyagglutinability, which is characterized by thrombocytopenia, leukopenia, and hemolytic anemia, but not nephritis $(26,44-46)$. The Tn antigen on the surfaces 
Table 2

Sequences of primers used for real time RT-PCR

\begin{tabular}{|c|c|c|}
\hline Genes & Sense $5^{\prime}-3^{\prime}$ & Antisense $5^{\prime}-3^{\prime}$ \\
\hline GalNAcT2 & AGGTCCTGACCTTCCTGGAC & TCСTACTTCCCCAGTTCTTCA \\
\hline C1GalT1 & TCATGCAAGGCATTCAGATG & ATGGGTTCTTCAGGGTCGTA \\
\hline Cosmc & GCTTTCCTGTCCCCAAGC & TGCTTTGTCACAGTGTTTGGT \\
\hline ST6GalNAcl & GCAACCACAGCCAAGACGCTCATTCCCAA & TGTCACGACCTTCTGCACCAAGGAGTAG \\
\hline ST6GalNAcll & AAGCTGCTACATCCGGACTTCA & GGGACAGATCGTGGTTTGCATA \\
\hline ST6GalNAcIV & CTGCAGCTCACCAGGATGTA & AACACGATGGGCCTCTTCT \\
\hline ST6GalNAcVI & GCCACCAGTGTGTGATTGTC & TGCTCAACCACGAATGAGAC \\
\hline IgA a chain & CCCCGACCAGCCCCAAGGTCT & GGCAGGACACTGGAACACGCTGTA \\
\hline J chain & CCCAGAGCAATATCTGTGATGA & GGTGGCAGGGAGTTGGTTTTAC \\
\hline$\beta$-actin & RDP-38-025 (R\&D) & RDP-38-025 (R\&D) \\
\hline
\end{tabular}

analogous situation has been shown for localization of ST6GalNAcI; its presence throughout the Golgi stacks explains its ability to override core $1 /$ core 2 pathways of O-linked glycosylation in breast tumor cells to produce sialylated GalNAc (Tn antigen) (48). The mechanism appears to include direct competition of ST6GalNAcI with C1GalT1, the first enzyme involved in the elongation of $O$-glycan chains (49, 50). These findings support the contention that the premature sialylation is probably a mechanism for the genesis of the Gal deficiency of the IgA1 O-linked glycans in IgAN (10).

of the blood cells is recognized by naturally occurring circulating antibodies but differs from the IgAN epitope in that it is composed of 3 adjacent GalNAc residues (47). In addition to, or perhaps independent of, the lower activity or expression of $\beta 1,3$-galactosyltransferase, early sialylation or oversialylation of GalNAc residues in the $O$-linked hinge-region glycans may contribute to the development of the Gal deficiency of IgA1 in patients with IgAN. If NeuAc is linked to GalNAc prior to attachment of Gal, this "premature" sialylation precludes subsequent galactosylation (32).

The activities of the enzymes that add carbohydrate residues to the $O$-linked glycans of the IgA1 hinge region were out of balance in the IgAN-IgA1S cell lines. The mean activity of $\beta 1,3$-galactosyltransferase in these cell lines was several-fold lower than that in the HC-IgA1S cell lines; in contrast, the mean activity of ST6GalNAc was several-fold higher in the HC-IgA1S cell lines. These differences in enzymatic activities correlated with the differences in transcription of the corresponding genes. Using BADG, an inhibitor of $\beta 1,3-$ galactosyltransferase, we confirmed that $\beta 1,3$-galactosyltransferase was active in the IgAN-IgA1S as well as the HC-IgA1S cell lines. The cell lines treated with BADG secreted IgA1 that had less Gal. The $O$-linked glycans on these IgA1 molecules contained sialylated and terminal GalNAc. We did not detect expression of ST6GalNAcI in any cell line, in agreement with our earlier observations (28). The expression of control genes, sialyltransferases ST6GalNAcIV and -VI, did not differ between the IgAN-IgA1S and HC-IgA1S cell lines nor did the transcript levels of GalNAcT2, IgA $\alpha$ chain, and J chain differ.

Immunofluorescence analyses demonstrated intracellular expression of the aberrantly glycosylated IgA1 in the IgAN-IgA1S cell lines but not in the HC-IgA1S cell lines. Confocal microscopy analysis localized the Gal-deficient IgA1 to the Golgi apparatus and showed that IgA1 contained sialylated GalNAc throughout the organelle, suggesting an early sialylation of hinge-region GalNAc residues. An

\section{Figure 7}

Gene transcriptional levels in IgA1-producing cell lines from IgAN patients and healthy controls. (A) Gene transcriptional levels of specific glycosyltransferases were quantitated by real-time RT-PCR, normalized to $\beta$-actin, and compared between patients $(n=11$; black bars) and controls ( $n=11$; white bars) using the E-method (55) as mean relative expression (values for controls were set to 1.0). Transcription levels of C1GalT1 and its molecular chaperone Cosmc were lower in IgAN-IgA1S cell lines, while that of ST6GalNAc/l was higher. (B) Gene expression of $J$ chain and $\alpha$ chain was similar in the IgAN$\operatorname{lgA} 1 \mathrm{~S}$ and $\mathrm{HC}$-lgA1S cell lines. Data are presented as mean $\pm \mathrm{SD}$. ${ }^{*} P<0.01 ;{ }^{*} P<0.001$.
In summary, we have successfully immortalized and cloned B cells that synthesized and secreted an undergalactosylated form of IgA1 that is characteristically found in the circulation and in the glomerular deposits of subjects with IgAN. This glycosylation aberrancy was due to complex changes in expression of specific glycosyltransferases with reduced expression of galactosyltransferase/Cosmc and elevated expression of sialyltransferase (Figure 9). These cell lines represent a new model for studies of mechanisms leading to aberrant IgA1 glycosylation in IgAN. They may be valuable in the development of new therapeutic strategies for IgAN, such as the use of specific inhibitors of ST6GalNAcII that would result in the generation of $O$-glycans that would not be sialylated and would thus remain accessible for normal galactosylation.

\section{Methods}

Human subjects. Peripheral blood was collected from 57 patients with biopsyproven IgAN (mean age, $39.7 \pm 15.0$ years; serum creatinine, $1.6 \pm 1.3 \mathrm{mg} / \mathrm{dl}$; urinary protein/creatinine ratio, $1.09 \pm 1.48$ ), from 63 healthy controls (mean age, $40.4 \pm 16.6$ years; serum creatinine, $1.0 \pm 0.3 \mathrm{mg} / \mathrm{dl}$; urinary pro-

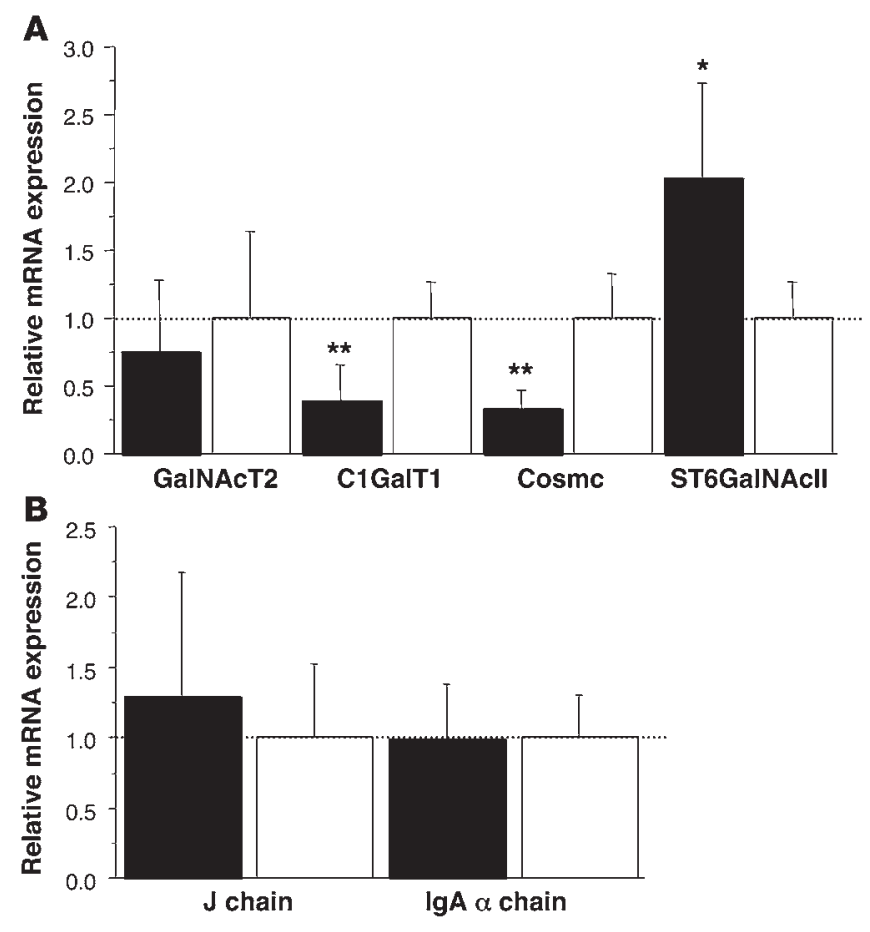




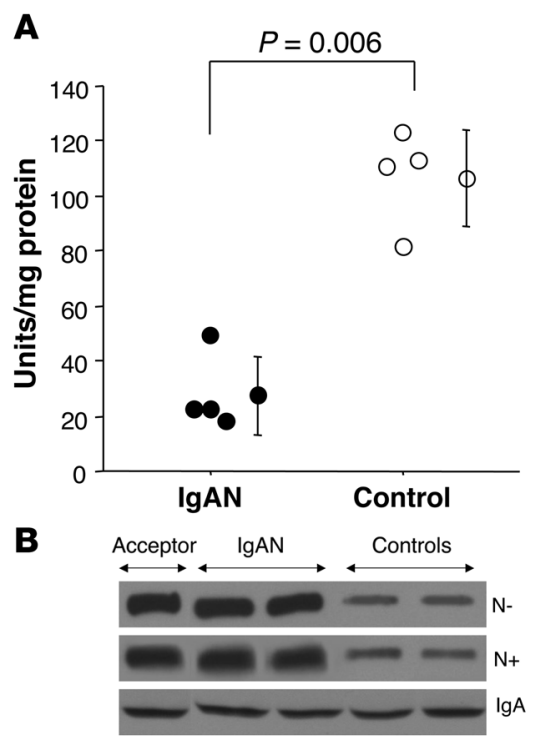

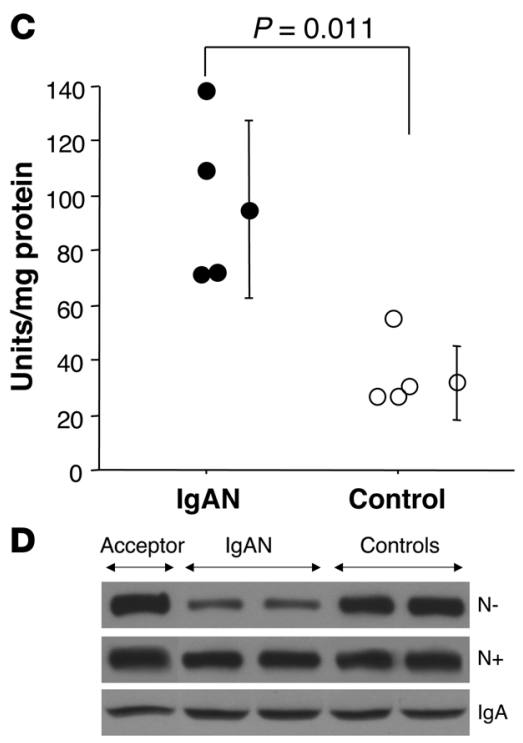

\section{Figure 8}

Enzyme activities of $\beta 1,3$-galactosyltransferase and ST6GalNAc in IgA1-producing cell lines. (A) $\beta 1,3-$ galactosyltransferase activity in Golgi-enriched fractions from IgAN-IgA1S cell lines was lower compared with that from $\mathrm{HC}$-IgA1S cell lines $(P=0.006)$. (B) HAA Western blotting confirmed that HAA reactivity of the acceptor $\operatorname{Ig} A 1$ decreased due to attachment of Gal to GalNAc, irrespective of neuraminidase treatment. (C) ST6GalNAc activity in Golgi-enriched fraction from IgAN-IgA1S cell lines was higher than that in the HC-IgA1S cell lines $(P=0.011)$. (D) HAA-Western blotting confirmed that $\mathrm{HAA}$ reactivity of the acceptor IgA1 decreased due to attachment of NeuAc to GalNAc (HAA reactivity increased after treatment with neuraminidase). $\mathrm{N}+$, neuraminidase treated; $\mathrm{N}-$, not treated with neuraminidase; $\lg \mathrm{A}$, load control developed with anti-IgA $\alpha$ chain-specific antibody. tein/creatinine ratio, $0.08 \pm 0.05$ ), and from 10 diseased controls (patients with biopsy-proven lupus nephritis; mean age, $38.1 \pm 12.1$ years; serum creatinine, $1.1 \pm 0.3 \mathrm{mg} / \mathrm{dl}$; urinary protein/creatinine ratio, $4.1 \pm 5.3$ ) for isolation of PBMCs. The IgAN patients included 34 white males and 16 white females, 1 African American male and 2 African American females, and 2 Asian males (Chinese, Bangladeshi) and 2 Asian females (Chinese, Filipina). One IgAN patient was on immunosuppressive therapy (corticosteroids). The healthy control group consisted of 25 white males and 33 white females, 1 African American male and 4 African American females. Each healthy control had no microscopic hematuria and a normal urinary protein/creatinine ratio or dipstick test for protein. Disease controls consisted of a group of 3 white females and 7 African American females. We determined the levels of IgA and Gal-deficient IgA1 in the urine samples from the $22 \operatorname{IgAN}$ and healthy control subjects by capture ELISA and expressed the data relative to urinary creatinine concentration (51). The findings were confirmed by Western blotting with $\alpha$ chain-specific antibody $(52,53)$. The levels of urinary IgA and Gal-deficient IgA1 were higher in the IgAN patients than in the healthy controls $(P<0.05$ and $P<0.01$, respectively; Table 1$)$. This observation is consistent with an ongoing renal injury in IgAN patients, as manifested clinically by proteinuria $(P<0.01)$.

The Institutional Review Boards at the University of Alabama at Birmingham and the University of Tennessee Health Sciences Center approved this study. Informed written consent was obtained from all adults and from a parent or legally authorized representative for all children; children age 8 years or older provided signed assent.

Isolation of PBMCs, transformation with EBV, and cloning of IgA1-secreting cell lines. PBMCs from patients with IgAN and lupus nephritis and healthy controls were isolated from heparinized peripheral blood by Ficoll-Hypaque density gradient. The B cell population was enriched from the PBMCs by removal of adherent cells through incubation in a plastic tissue-culture flask for 1 hour at $37^{\circ} \mathrm{C}$ and removal of T cells by CD3 (panT) Dynabeads, according to the manufacturer's instructions (Dynal AS). An alternative protocol for isolation of IgA surface-positive B cells (CD19 surface positive) included immunofluorescence surface staining followed by cell sorting. PBMCs from 11 randomly selected IgAN patients (IgAN-IgA1S) (8 white males, 1 African American male, and 2 white females; 4 subjects had microscopic hematuria at the time of study), 11 randomly selected white healthy controls (HC-IgA1S) (4 males), and purified IgA-positive cells from 3 of the 10 randomly selected patients with lupus nephritis (1 white and 2 African Americans) (Table 1) were then immortalized with EBV (54) in the General Clinical Research Center Laboratory Core Facility at the University of Alabama at Birmingham. IgA-positive cells from initial immortalized cultures of all 3 groups were isolated using biotin-labeled anti-IgA antibody and streptavidin-coated magnetic beads (CELLection Biotin Binder Kit; Invitrogen Dynal AS).

To establish cell lines from the initial EBV-immortalized PBMCs from patients with IgAN and healthy controls, we subcloned IgA1-secreting

Table 3

Incubation of cell lines from 5 IgAN patients and 5 healthy controls with BADG, an inhibitor of $\beta 1,3$-galactosyltransferase, augments Gal deficiency of the secreted $\lg A 1$

\section{$\lg$ A production}

$(\mu \mathrm{g} / \mathrm{ml})$

IgAN

Control
$4.2(3.5-7.9)$ $4.5(3.2-6.5)$
Sham treated

$\mathrm{N}+$

$(\%)^{\mathrm{A}}$

$31.5(29.3-45.0)^{B, C}$

$9.0(3.8-14.4)^{B, E}$

\section{BADG treated \\ $\mathrm{N}+$}

$(\%)^{\mathrm{A}}$

$90.4(87.5-98.1)^{\mathrm{C}}$

$92.2(86.4-97.7)^{\mathrm{E}}$
BADG treated

N-

$(\%)^{\mathrm{A}}$

$55.0(47.7-57.8)^{\mathrm{D}}$

$84.5(84.2-92.8)^{\mathrm{D}}$

Data are expressed as median and range. $P$ values are calculated by Mann-Whitney test. ${ }^{A}$ Ratio of HAA-reactive IgA1 to total IgA1 relative to standard Galdeficient neuraminidase-treated $(\mathrm{N}+)$ IgA1 (Mce) myeloma protein that was set as $100 \%$. ${ }^{\mathrm{B} P}<0.01$; comparison between sham-treated neuraminidase-treated cells from IgAN patients and healthy controls. ${ }^{C} P<0.01$, comparison between BADG-treated neuraminidase-treated and sham-treated neuraminidase-treated cells for IgAN patients. ${ }^{D} P<0.01$; comparison between cells from IgAN patients and healthy controls, both BADG-treated but not treated with neuraminidase $(\mathrm{N}-)$. ${ }^{\mathrm{E} P}<0.01$; comparison between BADG-treated neuraminidase-treated and sham-treated neuraminidase-treated cells for healthy controls. 


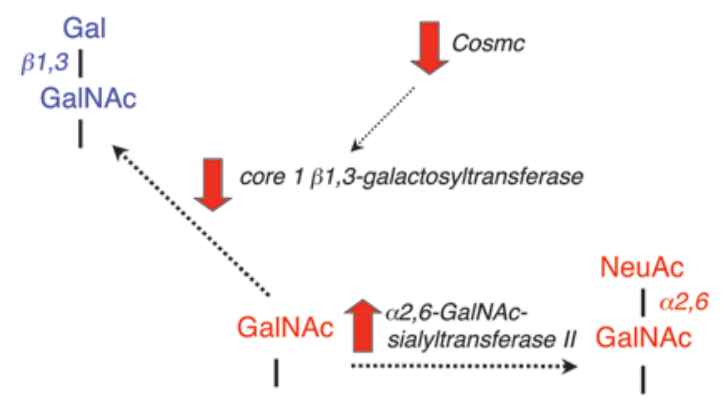

Figure 9

Complex changes in biosynthetic pathways of $O$-linked glycans in IgA1producing cell lines from IgAN patients. Transcription of C1GalT1 and Cosmc and enzyme activity of C1GalT1 was lower in the IgAN-IgA1S cell lines than in the HC-IgA1S cell lines, whereas the transcription and enzymatic activity of ST6GalNAcll was higher. Red arrows in front of enzyme names denote increased or decreased enzymatic expression/activity in the cell lines from IgAN patients compared with that in cell lines from healthy controls. Gal-containing glycans are shown in blue, Gal-deficient glycans, in red.

cell lines by limiting dilution (using 96-well plates seeded with 5 to 10 cells per well) in RPMI 1640 supplemented with L-glutamine, 20\% FCS, penicillin, and streptomycin. After several rounds of cloning and screening, IgA1-producing cell lines from all 11 IgAN patients and all 11 healthy controls were generated.

Measurement of immunoglobulin levels. The isotypes of the immunoglobulins secreted by the immortalized cells were determined by capture ELISA $(12,40)$. ELISA plates were coated with $\mathrm{F}\left(\mathrm{ab}^{\prime}\right)_{2}$ fragment of goat IgG antihuman IgA, IgG, or IgM (Jackson ImmunoResearch Inc.) at $1 \mu \mathrm{g} / \mathrm{ml}$. The bound immunoglobulins were then detected with biotin-labeled $\mathrm{F}\left(\mathrm{ab}^{\prime}\right)_{2}$ fragment of goat IgG anti-human IgA, IgG, or IgM antibody (Biosource), as appropriate. The binding was measured after addition of avidin-horseradish peroxidase conjugate (ExtrAvidin; Sigma-Aldrich), and the reaction was developed with the peroxidase chromogenic substrate o-phenylenediamine- $\mathrm{H}_{2} \mathrm{O}_{2}\left(\mathrm{OPD}-\mathrm{H}_{2} \mathrm{O}_{2}\right)$ (Sigma-Aldrich). The color reaction was stopped with $1 \mathrm{M}$ sulfuric acid, and the absorbance at $490 \mathrm{~nm}$ was measured using an EL312 BioKinetics microplate reader (BioTek Instruments Inc.).

Measurement of level of Gal-deficient IgA1. For capture ELISA, Costar 96-well U-bottom plates (Corning Inc.) were coated overnight at $4{ }^{\circ} \mathrm{C}$ with $\mathrm{F}\left(\mathrm{ab}^{\prime}\right)_{2}$ fragment of goat anti-human IgA (Jackson ImmunoResearch) at a concentration of $3 \mu \mathrm{g} / \mathrm{ml}$. Plates were blocked overnight at $4{ }^{\circ} \mathrm{C}$ with $2 \%$ BSA (Sigma-Aldrich) in PBS containing $0.05 \%$ Tween 20 $(\mathrm{v} / \mathrm{v})$. Samples diluted in blocking buffer were added to each well and incubated overnight at $4^{\circ} \mathrm{C}$. The captured IgA was subsequently desialylated by treatment for 3 hours at $37^{\circ} \mathrm{C}$ with $10 \mathrm{mU} / \mathrm{ml}$ neuraminidase from Vibrio cholerae (Roche) in $10 \mathrm{mM}$ sodium acetate buffer, $\mathrm{pH}=5$ (11). To assess the proportion of molecules with sialylated and terminal GalNAc residues, samples were analyzed in parallel, with and without neuraminidase treatment. Samples were then incubated for 3 hours at $37^{\circ} \mathrm{C}$ with GalNAc-specific biotinylated HAA lectin (Sigma-Aldrich) diluted 1:500 in blocking buffer $(35,40)$. The bound lectin was detected with avidin-horseradish peroxidase conjugate, and the reaction was developed as described above. The HAA reactivity of IgA1 in each sample was then calculated as OD units/ $1 \mu \mathrm{g}$ of IgA1. Naturally Gal-deficient IgA1 (Mce) purified from the plasma of a patient with IgA1 multiple myeloma $(12,35)$ was treated with neuraminidase and used as the standard. Thus, for comparisons of HAA binding, the OD units per $1 \mu \mathrm{g}$ neuraminidasetreated IgA1 (Mce) were assigned a value of $100 \%$.
Immunostaining of IgA1-secreting cell lines. Cells were cytospun on a glass microscopic slide, fixed with $4 \%$ paraformaldehyde (for surface staining) or acidic alcohol (for intracellular staining), and blocked with BSA (2\%) in PBS. Each specimen was stained with FITC-conjugated anti-human IgA antibody (Southern Biotech) and biotin-labeled HAA (Sigma-Aldrich), followed by TRITC-conjugated streptavidin (Southern Biotech). Cell nuclei were stained with Hoechst 33342 (Calbiochem). In a separate experiment, IgA-producing cells were also stained with FITC-conjugated anti-human IgA1 antibody (Southern Biotech) and PE-conjugated IgA2 (Southern Biotech) to determine the subclass of IgA.

The Golgi apparatus of the cells was stained with anti-human Golgin 97 mouse IgG followed by Alexa Fluor 488 goat anti-mouse IgG (Molecular Probes). For detecting IgA, biotin-labeled goat $\mathrm{F}\left(\mathrm{ab}^{\prime}\right)_{2}$ anti-human IgA antibodies (Biosource) and allophycocyanin-conjugated streptavidin (Southern Biotech) were used. Gal-deficient IgA1 was stained with biotin-labeled HAA and TRITC-conjugated streptavidin with or without neuraminidase treatment. Cell nuclei were stained with Hoechst 33342 reagent.

Confocal microscopy. Imaging was performed using a Leica DMIRBE inverted epifluorescence/Nomarski microscope outfitted with Leica TCS NT Laser Confocal optics (Leica Inc.) equipped with UV, argon, krypton, and helium/neon lasers. An image analysis program (IPLab, version 3.6; BD Biosciences) was used to examine the colocalization of proteins by measuring the line intensity. The intensity profile within the line drawn through the diameter of the cell, including the Golgi apparatus, demonstrated the spatial relationship of the corresponding proteins visualized in the graph.

Size-exclusion chromatography. To determine the molecular form of IgA1 secreted by the IgAN-IgA1S and HC-IgA1S cell lines, $1 \mathrm{ml}$ culture supernatant was filtered through a $0.45-\mu \mathrm{m}$ filter (Pall Corp.) and fractionated on a calibrated Superose 6 column $(600 \times 12 \mathrm{~mm})$ equilibrated in PBS $(12,20)$. The fractions were analyzed by ELISA for IgA and Gal-deficient IgA1. Purified polymeric and monomeric IgA1 myeloma proteins were used as molecular mass standards.

SDS-PAGE and Western blotting. Serum and culture supernatants were separated by SDS-PAGE using $4 \%-20 \%$ gradient slab gels (Bio-Rad) under reducing or nonreducing conditions. Protein levels of the loaded samples were normalized to IgA concentration. The gels were blotted to PVDF membranes and incubated with antibody specific for IgA $\alpha$ chains (Vector Laboratories) or biotin-labeled lectins specific for terminal GalNAc (HAA; Sigma-Aldrich) or $\alpha 2,6$-bound NeuAc (SNA; EY Laboratories Inc.). HAA reacts with terminal GalNAc but not with sialylated GalNAc or GalNAcGal disaccharide. Reaction of HAA with a terminal GalNAc is blocked by an attached NeuAc; therefore, the blots were treated with neuraminidase from $V$. cholerae (Roche) on PVDF membranes for 3 hours or overnight before the lectin binding (40). The visualization of positive bands was accomplished by subsequent incubation of the membrane with avidin-peroxidase conjugate, followed by enhanced chemiluminescence detection (Pierce) (40).

Real-time RT-PCR. Total RNA was isolated using RNAStat60 (13) from $1 \times 10^{6}$ cells and treated with DNase. mRNA was converted to cDNA using SuperScript II reverse transcriptase (Invitrogen). Real-time RT-PCR was performed using LightCycler FastStart DNA Master SYBR Green I chemistry as suggested by the manufacturer (Roche) with $\mathrm{MgCl}_{2}$ adjusted to a final concentration of $2.6 \mathrm{mM}$. Primers used for amplification of GalNAcT2, C1GalT1, Cosmc, ST6GalNAcI, -II, -IV, and -VI, IgA $\alpha$ chain, and $J$ chain are listed in Table 2. Primers for $\beta$-actin amplification (housekeeping gene) were purchased from R\&D Systems. Real-time RT-PCR was performed for 42 cycles of denaturation at $95^{\circ} \mathrm{C}$, annealing at $60^{\circ} \mathrm{C}$, and extension at $72^{\circ} \mathrm{C}$. Specificity of primers was verified by melting-point analysis and by gel electrophoresis of the amplicons. Data comparisons were performed by the E-method (55) and calculated as mean expression in IgAN-IgA1S cell lines relative to the expression in HC-IgA1S cell lines. 
Preparation of the Golgi-enriched fraction from IgA1-producing cell lines. Golgienriched cellular fractions were prepared using a published method (28). In brief, cells $\left(1 \times 10^{7}\right)$ were harvested and homogenized on ice in a PotterElvehjem homogenizer in $2 \mathrm{ml}, 25 \mathrm{mM}, 2$-[ $N$-morpholino]ethanesulfonic acid (MES) buffer ( $\mathrm{pH}$ 6.5) containing $75 \mathrm{mM} \mathrm{NaCl}$ and $1 \times$ Complete Mini EDTA-free protease inhibitor mix (Roche). The homogenate was centrifuged at $1,000 \mathrm{~g}$ for 10 minutes. The supernatant was recentrifuged at $27,000 \mathrm{~g}$ for 1 hour, and the pellet was resuspended in $25 \mathrm{mM}$ MES buffer, $\mathrm{pH}$ 6.5, containing $75 \mathrm{mM} \mathrm{NaCl}, 10 \mathrm{mM} \mathrm{MnCl}_{2}, 10 \%$ glycerol (v/v), 1\% Triton X-100, and $1 \times$ Complete Mini EDTA-free protease inhibitor mix (Roche). After 1 hour, the suspension was centrifuged at $100,000 \mathrm{~g}$ for 1 hour and the supernatant, designated as the Golgi-enriched fraction, was used as the source of the glycosyltransferases. All purification steps were performed at $4^{\circ} \mathrm{C}$.

Preparation of desialylated Gal-deficient IgA1. IgA1 (Mce) myeloma protein $(500 \mu \mathrm{g})$ was desialylated with $30 \mathrm{mU}$ neuraminidase from $V$. cholerae (Roche) in $50 \mathrm{mM}$ sodium acetate buffer $\mathrm{pH} 5.5$ at $37^{\circ} \mathrm{C}$ for 12 hours. The desialylated Gal-deficient IgA1 was purified by size-exclusion chromatography (28) and used as acceptor in the sialyltransferase and galactosyltransferase assays.

Measurement of $\beta 1,3$-galactosyltransferase and ST6GalNAc enzyme activities. To measure enzymatic activity of $\beta 1,3$-galactosyltransferase, we used a modification of previously published methods $(24,56)$. In brief, the galactosyltransferase activity was determined using desialylated Gal-deficient IgA1 (Mce) myeloma protein as the acceptor and UDP-Gal (Sigma-Aldrich) as the donor substrate. Desialylated IgA1 (Mce) myeloma protein $(2 \mu \mathrm{g})$ was incubated with $10 \mu \mathrm{l} \mathrm{Golgi-enriched} \mathrm{fraction} \mathrm{for} 4$ hours at $37^{\circ} \mathrm{C}$ in $45 \mu \mathrm{l}$ reaction buffer (100 mM MES buffer, pH 6.5; $20 \mathrm{mM} \mathrm{MnCl}_{2}, 0.4 \%$ Triton X-100, $2 \mathrm{mM}$ ATP, 0.4 mM UDP-Gal, and $1 \times$ Complete Mini EDTA-free protease inhibitor $\mathrm{mix})$. The reaction was stopped by snap-freezing the samples at $-80^{\circ} \mathrm{C}$.

Enzymatic activity of ST6GalNAc was determined as described earlier, with a few modifications $(28,56)$. In brief, the sialyltransferase activity was determined using desialylated Gal-deficient IgA1 (Mce) myeloma protein as the acceptor and CMP-NeuAc (Sigma-Aldrich) as the donor substrate. Desialylated IgA1 (Mce) myeloma protein $(2 \mu \mathrm{g})$ was incubated with $10 \mu \mathrm{l}$ Golgi-enriched fraction for 4 hours at $37^{\circ} \mathrm{C}$ in $30 \mu \mathrm{l}$ reaction buffer $(50 \mathrm{mM}$ MES buffer, pH 6.5; $2 \mathrm{mM} \mathrm{CaCl}_{2}, 2 \mathrm{mM} \mathrm{MnCl}_{2}, 10 \mathrm{mM} \mathrm{MgCl}_{2}, 0.4 \%$ Triton $\mathrm{X}$-100, 10 mM CMP-NeuAc, 1 mM 2,3-dehydro-2-deoxy-Neu5Ac [sialidase inhibitor, Sigma-Aldrich]; $1 \times$ Complete Mini EDTA-free protease inhibitor $\mathrm{mix})$. The reaction was stopped by snap-freezing the samples at $-80^{\circ} \mathrm{C}$.
The amount of Gal or NeuAc added to GalNAc residues on IgA1 was determined based on changes in the HAA lectin binding to IgA1 before and after the sialyltransferase reaction (28). Specificity of the assay was verified in a parallel reaction of HAA with the IgA1 in the reaction mixture that was treated with neuraminidase after capture on the ELISA plate. One unit of the enzyme activity was defined as a decrease of HAA binding to IgA1 by $1.0 \mathrm{OD}$ in 4 hours. The enzyme activity was normalized per mg protein in the Golgi-enriched preparation in the reaction mixture.

Inbibition of galactosyltransferase. BADG was used as inhibitor of $\beta 1,3$-galactosyltransferase (36). IgA1-secreting cell lines $\left(1 \times 10^{6}\right.$ cells) from IgAN patients and controls were cultured with or without $5 \mathrm{mM}$ BADG dissolved with $3.7 \%$ methanol in RPMI 1640 medium supplemented with L-glutamine, $20 \%$ FCS, penicillin, and streptomycin. After 72 hours, levels of IgA1 and HAA-reactive IgA1 produced by the cell lines were measured by ELISA.

Statistics. Correlations between the different parameters were analyzed by Student's $t$ test, 2 tailed, or by the Mann-Whitney test. ANOVA was used to determine differences in the characteristics among multiple groups. Data were expressed as mean \pm SD or median values. $P$ values of less than 0.05 were considered significant. All statistical analyses were performed with StatView 5.0 software (Abacus Concept Inc.).

\section{Acknowledgments}

This work was supported by grants from the NIH (DK78244, DK61525, AR49084, DK71802, DK47322, and DK64400), the General Clinical Research Centers of the University of Alabama at Birmingham (M01 RR00032) and the University of Tennessee Health Sciences Center (M01 RR00211), and the Czech Republic (VZMSM0021620812 and KJ586206106). The authors appreciate the critical reading of the manuscript by Fiona Hunter.

Received for publication July 3, 2007, and accepted in revised form October 24, 2007.

Address correspondence to: Jan Novak, Department of Microbiology, University of Alabama at Birmingham, 84519 th Street South, BBRB 734, Birmingham, Alabama 35294, USA. Phone: (205) 934-4480; Fax: (205) 934-3894; E-mail: jannovak@uab.edu.
1. Julian, B.A., Waldo, F.B., Rifai, A., and Mestecky, J. 1988. IgA nephropathy, the most common glomerulonephritis worldwide. A neglected disease in the United States? Am. J. Med. 84:129-132.

2. Emancipator, S.N., Mestecky, J., and Lamm, M.E. 2005. IgA nephropathy and related diseases. In Mucosal immunology. 3rd edition. J. Mestecky, et al., editors. Elsevier. Amsterdam, The Netherlands. 1579-1600.

3. Jennette, J.C. 1988. The immunohistology of IgA nephropathy. Am. J. Kidney Dis. 12:348-352.

4. Czerkinsky, C., et al. 1986. Circulating immune complexes and immunoglobulin A rheumatoid factor in patients with mesangial immunoglobulin A nephropathies. J. Clin. Invest. 77:1931-1938.

5. Allen, A.C., et al. 2001. Mesangial IgA1 in IgA nephropathy exhibits aberrant $O$-glycosylation: Observations in three patients. Kidney Int. 60:969-973

6. Hiki, Y., et al. 2001. Mass spectrometry proves under-O-glycosylation of glomerular IgA1 in IgA nephropathy. Kidney Int. 59:1077-1085.

7. Barratt, J., and Feehally, J. 2005. IgA nephropathy. J. Am. Soc. Nephrol. 16:2088-2097.

8. Coppo, R., and Amore, A. 2004. Aberrant glycosylation in $\operatorname{IgA}$ nephropathy (IgAN). Kidney Int. 65:1544-1547.
9. Julian, B.A., and Novak, J. 2004. IgA nephropathy: an update. Curr. Opin. Nephrol. Hypertens. 13:171-179.

10. Novak, J., Julian, B.A., Tomana, M., and Mestecky, J. 2001. Progress in molecular and genetic studies of IgA nephropathy. J. Clin. Immunol. 21:310-327.

11. Tomana, M., et al. 1997. Galactose-deficient IgA1 in sera of IgA nephropathy patients is present in complexes with IgG. Kidney Int. 52:509-516.

12. Tomana, M., et al. 1999. Circulating immune complexes in IgA nephropathy consist of IgA1 with galactose-deficient hinge region and antiglycan antibodies. J. Clin. Invest. 104:73-81.

13. Novak, J., et al. 2002. Interactions of human mesangial cells with IgA and IgA-containing circulating immune complexes. Kidney Int. 62:465-475.

14. Gomez-Guerrero, C., Gonzalez, E., Hernando, P., Ruiz-Ortega, M., and Egido, J. 1993. Interaction of mesangial cells with IgA and IgG immune complexes: a possible mechanism of glomerular injury in IgA nephropathy. Contrib. Nephrol. 104:127-137.

15. Gomez-Guerrero, C., Lopez-Armada, M.J., Gonzalez, E., and Egido, J. 1994. Soluble IgA and IgG aggregates are catabolized by cultured rat mesangial cells and induce production of TNF- $\alpha$ and IL- 6 , and proliferation. J. Immunol. 153:5247-5255.

16. Lopez-Armada, M.J., Gomez-Guerrero, C., and Egido, J. 1996. Receptors for immune complexes activate gene expression and synthesis of matrix proteins in cultured rat and human mesangial cells: role of TGF- $\beta$. J. Immunol. 157:2136-2142.

17. Duque, N., Gomez-Guerrero, C., and Egido, J. 1997. Interaction of IgA with Fc $\alpha$ receptors of human mesangial cells activates transcription factor nuclear factor-kB and induces expression and synthesis of monocyte chemoattractant protein1, IL-8, and IFN-inducible protein 10. J. Immunol. 159:3474-3482.

18. Chen, A., Chen, W.P., Sheu, L.F., and Lin, C.Y. 1994. Pathogenesis of IgA nephropathy: in vitro activation of human mesangial cells by IgA immune complex leads to cytokine secretion. J. Pathol. 173:119-126.

19. Amore, A., et al. 2001. Glycosylation of circulating IgA in patients with IgA nephropathy modulates proliferation and apoptosis of mesangial cells. J. Am. Soc. Nephrol. 12:1862-1871.

20. Novak, J., et al. 2005. IgA1-containing immune complexes in IgA nephropathy differentially affect proliferation of mesangial cells. Kidney Int. 67:504-513.

21. Moura, I.C., et al. 2004. Glycosylation and size of IgA1 are essential for interaction with mesangial transferrin receptor in IgA nephropathy. J. Am. Soc. Nephrol. 15:622-634.

22. Moura, I.C., et al. 2005. Engagement of transferrin receptor by polymeric IgA1: evidence for a posi- 
tive feedback loop involving increased receptor expression and mesangial cell proliferation in IgA nephropathy. J. Am. Soc. Nephrol. 16:2667-2676.

23. Iwasaki, H., et al. 2003. Initiation of $\mathrm{O}$-glycan synthesis in IgA1 hinge region is determined by a single enzyme, UDP-N-acetyl- $\alpha$-D-galactosamine: polypeptide $N$-acetylgalactosaminyltransferase 2 . J. Biol. Chem. 278:5613-5621.

24. Ju, T., Brewer, K., D’Souza, A., Cummings, R.D., and Canfield, W.M. 2002. Cloning and expression of human core $1 \beta 1,3$-galactosyltransferase. J. Biol. Chem. 277:178-186.

25. Ju, T., and Cummings, R.D. 2002. A unique molecular chaperone Cosmc required for activity of the mammalian core $1 \beta 3$-galactosyltransferase. Proc. Natl. Acad. Sci. U. S. A. 99:16613-16618.

26. Ju, T., and Cummings, R.D. 2005. Protein glycosylation: chaperone mutation in Tn syndrome. Nature. 437:1252.

27. Ikehara, Y., et al. 1999. Cloning and expression of a human gene encoding an $\mathrm{N}$-acetylgalactosamine$\alpha 2,6$-sialyltransferase (ST6GalNAc I): a candidate for synthesis of cancer-associated sialyl-Tn antigens. Glycobiology. 9:1213-1224.

28. Raska, M., et al. 2007. Identification and characterization of CMP-NeuAc:GalNAc-IgA1 $\alpha 2,6$-sialyltransferase in IgA1-producing cells. J. Mol. Biol. 369:69-78.

29. Marcos, N.T., et al. 2004. Role of the human ST6GalNAc-I and ST6GalNAc-II in the synthesis of the cancer-associated sialyl-Tn antigen. Cancer Res. 64:7050-7057.

30. Gillespie, W., Kelm, S., and Paulson, J.C. 1992. Cloning and expression of the Gal $\beta 1,3$-GalNAc $\alpha 2,3$ sialyltransferase. J. Biol. Chem. 267:21004-21010.

31. Priatel, J.J., et al. 2000. The ST3Gal-I sialyltransferase controls CD8+ T lymphocyte homeostasis by modulating $O$-glycan biosynthesis. Immunity. 12:273-283.

32. Schachter, H., McGuire, E.J., and Roseman, S. 1971. Sialic acids. XIII. A uridine diphosphate D-galactose: mucin galactosyltransferase from porcine submaxillary gland. J. Biol. Chem. 246:5321-5328.

33. Peppard, J.V., Kaetzel, C.S., and Russell, M.W. 2005.
Phylogeny and comparative physiology of IgA. In Mucosal immunology. 3rd edition. J. Mestecky, et al., editors. Elsevier. Amsterdam, The Netherlands. 195-210.

34. Mestecky, J., Moro, I., Kerr, M.A., and Woof, J.M. 2005. Mucosal immunoglobulins. In Mucosal immunology. 3rd edition. J. Mestecky, et al., editors. Elsevier. Amsterdam, The Netherlands. 153-181.

35. Moldoveanu, Z., et al. 2007. Patients with IgA nephropathy have increased serum galactose-deficient IgA1 levels. Kidney Int. 71:1148-1154.

36. Gala, F.A., and Morrison, S.L. 2002. The role of constant region carbohydrate in the assembly and secretion of human IgD and IgA1. J. Biol. Chem. 277:29005-29011.

37. Mestecky, J., et al. 1993. Defective galactosylation and clearance of IgA1 molecules as a possible etiopathogenic factor in IgA nephropathy. Contrib. Nephrol. 104:172-182.

38. Kutteh, W.H., et al. 1983. Biosynthesis of J-chain in human lymphoid cells producing immunoglobulins of various isotypes. Mol. Immunol. 20:967-976.

39. Linossier, M.T., Palle, S., and Berthoux, F. 2003. Different glycosylation profile of serum IgA1 in IgA nephropathy according to the glomerular basement membrane thickness: normal versus thin. Am J. Kidney Dis. 41:558-564.

40. Moore, J.S., et al. 2007. Reactivities of $N$-acetylgalactosamine-specific lectins with human IgA1 proteins. Mol. Immunol. 44:2598-2604.

41. Leung, J.C., Tang, S.C., Chan, D.T., Lui, S.L., and Lai, K.N. 2002. Increased sialylation of polymeric lambda-IgA1 in patients with IgA nephropathy. J. Clin. Lab. Anal. 16:11-19.

42. Allen, A.C., Topham, P.S., Harper, S.J., and Feehally, J. 1997. Leucocyte $\beta 1,3$ galactosyltransferase activity in IgA nephropathy. Nephrol. Dial. Transplant. 12:701-706.

43. Qin, W., et al. 2005. Peripheral $\beta$ lymphocyte beta1,3-galactosyltransferase and chaperone expression in immunoglobulin A nephropathy. J. Intern. Med. 258:467-477.

44. Berger, E.G. 1999. Tn-syndrome. Biochim. Biophys. Acta. 1455:255-268.
45. Thurnher, M., Rusconi, S., and Berger, E.G. 1993. Persistent repression of a functional allele can be responsible for galactosyltransferase deficiency in Tn syndrome. J. Clin. Invest. 91:2103-2110.

46. Vainchenker, W., et al. 1985. Presence of the Tn antigen on hematopoietic progenitors from patients with the Tn syndrome. J. Clin. Invest. 75:541-546.

47. Nakada, H., et al. 1991. Elucidation of an essential structure recognized by an anti-GalNAca-Ser(Thr) monoclonal antibody (MLS 128). J. Biol. Chem. 266:12402-12405.

48. Sewell, R., et al. 2006. The ST6GalNAc-I sialyltransferase localizes throughout the Golgi and is responsible for the synthesis of the tumor-associated sialyl-Tn $O$-glycan in human breast cancer. J. Biol. Chem. 281:3586-3594.

49. Julien, S., et al. 2001. Expression of sialyl-Tn antigen in breast cancer cells transfected with the human CMP-Neu5Ac: GalNAc $\alpha 2,6$-sialyltransferase (ST6GalNAc I) cDNA. Glycoconj. J. 18:883-893.

50. Julien, S., et al. 2006. ST6GalNAc I expression in MDA-MB-231 breast cancer cells greatly modifies their $O$-glycosylation pattern and enhances their tumourigenicity. Glycobiology. 16:54-64.

51. Matousovic, K., et al. 2006. IgA1-containing immune complexes in the urine of IgA nephropathy patients. Nephrol. Dial. Transplant. 21:2478-2484.

52. Julian, B.A., et al. 2007. Electrophoretic methods for analysis of urinary polypeptides in IgA-associated renal diseases. Electrophoresis. 28:4469-4483.

53. Julian, B.A., et al. 2007. Urinary biomarkers of IgA nephropathy and other IgA-associated renal diseases. World J. Urol. 25:467-476.

54. Hajdu, I., Moldoveanu, Z., Cooper, M.D., and Mestecky, J. 1983. Ultrastructural studies of human lymphoid cells. $\mu$ and $J$ chain expression as a function of B cell differentiation. J. Exp. Med. 158:1993-2006.

55. Tellmann, G. 2006. The E-Method: a highly accurate technique for gene-expression analysis. Nat. Methods. 3:i-ii.

56. Basset, C., et al. 2000. Enhanced sialyltransferase activity in B lymphocytes from patients with primary Sjögren's syndrome. Scand. J. Immunol. 51:307-311. 